Note that the corresponding directed graph has the Hamiltonian circuit $1 \rightarrow 6 \rightarrow$ $5 \rightarrow 3 \rightarrow 4 \rightarrow 2 \rightarrow 7 \rightarrow 1$ and so is strongly connected. Hence, for no permutation $P$ does $P A P^{-1}$ reduce. Using the algorithm described in [3] one obtains the permutations $P=(1725643$ ) and $Q=(1)(236754)$. Applying $P$ and $Q$ to the rows and columns of $A$, one obtains:

$$
P A Q=\left|\begin{array}{cc:ccc:cc}
1 & 2 & 0 & 0 & 0 & 0 & 0 \\
1 & 4 & 0 & 0 & 0 & 0 & 0 \\
\hdashline 0 & 3 & 4 & 0 & 0 & 0 & 0 \\
3 & 0 & 2 & 2 & 0 & 0 & 0 \\
0 & 0 & 2 & 0 & 4 & 0 & 0 \\
\hdashline 3 & 0 & 0 & 3 & 0 & 1 & 2 \\
0 & 0 & 0 & 0 & 0 & 5 & 1
\end{array}\right|
$$

The authors are indebted to F. Harary for a pre-publication copy of his paper [4].

The University of Manitoba

Winnipeg, Canada

1. A. L. Dulmage \& N. S. Mendelsohn, "A structure theory of bipartite graphs of finite exterior dimension," Trans. Roy. Soc. Canada, Sect. III 53, 1959, p. 1-13.

2. D. M. Johnson, A. L. Dulmage, \& N. S. Mendelsohn, "Connectivity and reducibility of graphs", Canad.J. Math., 14, 1962, p. 529-539.

3. A. L. Dulmage \& N. S. Mendelsohn, "Two algorithms for bipartite graphs," to be published in Soc. Indust. Appl. Math.

4. F. HARARY, "A graph theoretic approach to matrix inversion by partitioning," to be published in Numer. Math.

5. M. HALL, "An algorithm for distinct representatives," Amer. Math. Monthly, 1956, p. $716-717$.

6. L. R. Ford \& D. R. Fulkerson, "A simple algorithm for finding maximal network flows and an application to the Hitchcock problem," Canad.J. Math. 9, 1957, p. 210-218. Also in Management Sci., October 1956, p. 24-32.

\title{
Missing Data Correlation Computations
}

\section{By R. I. Jennrich}

In correlation analysis or in any multivariate analysis based on the computation of a correlation or covariance matrix, the applied statistician often runs into the problem of missing data. To avoid complication in computing the correlation matrix, a complete observation vector is often discarded when only one or more of its components are missing. If a correlation matrix is computed by means of a standard electronic computer program, this procedure is often necessary. A large percentage of data may be thrown away when only a small percentage is missing. This note describes a modification in the standard computing scheme which eliminates this waste of data.

Let $x_{n 1}, x_{n 2}, \cdots, x_{n p}$ denote the $p$ components of the $n$th observation vector, $n=1,2, \cdots, N$. It is customary to add an $n+1$ st component to this vector which is identically equal to one. That is $x_{n, p+1}=1$. The cross product matrix

$$
a_{i j}=\sum_{n=1}^{N} x_{n i} x_{n j} \quad i=1, \cdots, p+1 ; j=1, \cdots, p+1
$$

Received September 7, 1961. 
is customarily computed and the correlation matrix is computed from this matrix by using the fact that

$$
a_{p+1, p+1}=N, a_{i, p+1}=\sum_{n=1}^{N} x_{n i} ; i=1, \cdots, p .
$$

In addition to adding a component which is identically one to each observation vector, let us form a new vector $c_{n 1}, c_{n 2}, \cdots, c_{n p}$ where $c_{n i}$ is zero if the $i$ th component of the observation vector is missing and one otherwise. Letting each element of missing data have value zero, we form the cross product matrices

$$
\begin{array}{rl}
s_{i j}=\sum_{n=1}^{N} x_{n i} x_{n j} & i, j=1, \cdots, p+1 \\
n_{i j}=\sum_{n=1}^{N} c_{n i} c_{n j} & i, j=1, \cdots, p .
\end{array}
$$

The means $m_{i}$, covariances $v_{i j}$, and correlations $r_{i j}$ are computed from these matrices by the formulas

$$
\begin{aligned}
m_{i} & =\frac{1}{n_{i i}} s_{i, p+1} \\
v_{i j} & =\frac{1}{n_{i j}} s_{i j}-m_{i} m_{j} \\
r_{i j} & =\frac{v_{i j}}{\sqrt{v_{i i}} \sqrt{v_{j j}}} .
\end{aligned}
$$

It should be noted that the statistical properties of these estimates will differ slightly from those computed without missing data. A discussion of some of these properties is given by S. S. Wilks [1].

A FORTRAN program for the computations described in this note is in use at the University of Wisconsin. A write-up and program deck can be obtained by writing to the author.

The University of Wisconsin Madison 6, Wisconsin

1. S. S. WiLKs, "Moments and distributions of estimates of population parameters from fragmentary samples," Ann. Math. Stat., v. 3, 1932, p. 163.

\section{Polynomial Approximations to $I_{0}(x), I_{1}(x)$ and Related Functions}

\section{By F. D. Burgoyne}

Hitchcock [1] gives polynomial approximations to some Bessel functions of order zero and one and to some related functions. Notable omissions from his list are any approximations to $I_{0}(x)$ or $I_{1}(x)$. The following approximations may serve to fill this gap.

If we write $I_{n}(x)=(2 \pi x)^{-1 / 2} e^{x} F_{n}(x)$, then with the maximum error stated in brackets in each case, and provided $0 \leqq t \leqq 1$,

Received August 16, 1961. 